Ilario Cola

Università di Łódź

https://doi.org/10.18778/8220-506-0.02

\title{
È POSSIBILE UNA DEFINIZIONE DEL CONCETTO DI ITALIANITÀ?
}

Riassunto: L'italianità è un concetto molto complesso e per questo la sua definizione non risulta di facile portata. In questo saggio gli autori cercano di evidenziare queste complessità e di fornire una possibile definizione di italianità che si distingua o completi quelle già esistenti. Ma prima di giungere a questa definizione occorre tuttavia tener conto anche dei falsi miti, idealità o pregiudizi che l'italianità si porta dietro sia in Italia che all'estero, tra gli italiani e gli stranieri, tra gli italofili e gli italici. L'articolo evidenzia quindi anche alcuni dati "scomodi" dell'italianità che normalmente vengono sottaciuti o poco evidenziati, riguardanti gli aspetti meno nobili della realtà italiana sia nel passato che nel presente. Essendo l'Italia un paese molto variegato, geograficamente, storicamente, culturalmente e linguisticamente, questa varietà ha prodotto anche delle differenze all'interno del Bel Paese con lacerazioni e forti contrasti. Parlare di italianità comporta anche di parlare di questi contrasti, "anormalità", a volte dei veri e propri paradossi, che non sono fenomeni moderni ma erano già stati registrati e riportati attraverso i secoli da italiani e stranieri autorevoli. Riascoltare queste voci insieme alla riproposizione di vicende storiche che gli italiani hanno vissuto permette agli autori di riflettere e di affrontare la realtà italiana attraverso un processo costante di analisi e sintesi, di avvicinamento e di estraniamento. Solo dopo questo processo di "purificazione" sarà possibile parlare con maggiore distensione di Italia, lingua italiana ed italiani come elementi costituenti dell'italianità.

Parole chiave: italianità, italicità, Italia, italiani, cultura italiana, lingua italiana, stereotipi, pregiudizi, definizione.

Abstract: Is it possible to define the concept of Italianity? Italianity is a very
complex concept and for this reason its definition is not easy. In this essay the authors
try to highlight these complexities and to provide a possible definition of Italianity that
distinguishes or completes existing ones. However, before reaching this definition it is
necessary to take into account also the false myths, ideals or prejudices that Italianity
carries with it both in Italy and abroad, between Italians and foreigners, between
Italophiles and Italics. Therefore, the article also highlights some "uncomfortable" data
of the Italian spirit that is normally underestimated or poorly highlighted, concerning 
the less noble aspects of the Italian reality both in the past and in the present. Since Italy is a very varied country, geographically, historically, culturally and linguistically, this variety has also produced differences within the Bel Paese with lacerations and strong contrasts. Talking about Italianity also involves talking about these contrasts, "abnormalities", sometimes real paradoxes, which are not modern phenomena but had already been recorded and reported through the centuries by authoritative Italians and foreigners. Listening to these voices together with the re-proposal of historical events that Italians have experienced allows the authors to reflect and face the Italian reality through a constant process of analysis and synthesis, of approach and estrangement. Only after this process of "purification" will it be possible to speak with greater détente about Italy, the Italian language and Italians as constituent elements of the Italian spirit.

Keywords: Italianity, Italicity, Italy, Italians, Italian culture, Italian language, prejudices, definition.

Ogni atto di creazione è,
prima di tutto,
un atto di distruzione.

Pablo Picasso

\section{Introduzione}

Siamo in grado di definire il concetto di italianità? Sembra difficile rispondere. Allora proviamo a riformulare la domanda, cercando di facilitare una risposta: è possibile contenere, descrivere e circoscrivere in poche righe o in alcune pagine, finanche in un libro intero, le caratteristiche tipiche geografiche, le peculiarità storiche, linguistiche e culturali di questo Paese chiamato Italia e del suo popolo, gli Italiani?

La risposta ci pare sempre e inesorabilmente incerta: è possibile certamente descrivere alcune caratteristiche geografiche, ma il territorio italiano è talmente diversificato che darne conto in senso schematico andrebbe a scapito proprio di alcune peculiarità meno note al grande pubblico. Citare troppo spesso le Dolomiti, le colline toscane, i faraglioni di Capri, la costiera amalfitana, la laguna di Venezia; oppure i monumenti di Roma o i vicoli di Napoli, l'Etna o il Vesuvio, ecc.; come esempio di italianità geografica rischia di non rendere sufficiente giustizia, nonché di oscurare altre realtà peculiari, come le Alpi liguri, il carsismo pugliese, i laghi vulcanici laziali, le isole Tremiti, il massiccio montuoso del Sirente-Velino, le valli di Comacchio, i vicoli di Genova, le dune di Piscinas e mille altri luoghi, più o meno noti, che fanno parte anch'essi 
dell'italianità. Stesso problema riguarderebbe il concentrarci esclusivamente su determinati fatti storici e/o culturali.

Insomma, definire l'italianità, tramite soliti schemi ripetitivi, potrebbe essere riduttivo se non addirittura scomodo, in quanto costruiscono un'immagine ideale, il che non significa che questa "italianità ideale" costituisca sempre un'immagine positiva: pensiamo a chi guardava o guarda all'Italia soltanto attraverso serie tv come La Piovra (1984-2001) o Gomorra (2014-.

Neanche gli esperti, né le fonti enciclopediche o lessicografiche, ci danno una riposta univoca, mettendo a confronto spesso due concetti vicini nella loro portata semantica e culturale: "italianità" e "italicità", che suggeriscono una nuova prospettiva sugli "Italiani" stessi, ma anche sugli "Italici". Se per i primi non si hanno dubbi nella loro categorizzazione nazionale d'origine legata al territorio d'Italia, ma anche fuori da questo territorio (gli Italiani all'estero), nel caso dei secondi si parla oggi di "soggetti sparsi per il mondo che dimostrano un interesse verso l'Italia, i suoi valori, la sua cultura, pur essendo nati all'estero e spesso senza conoscere la lingua italiana" (Bombi e Orioles 2011: 21-22). Come riportano gli autori, la comunità di questi "italici" è "in grado di svolgere un ruolo importante nel mondo e ridare slancio all'identità italiana” (ibid.).

Un chiaro significato del concetto di italicità in questa sua nuova veste semantica è dato finalmente dagli iniziatori del progetto Italici promosso dall'Associazione Globus et Locus: "Gli italici non sono tanto i cittadini italiani in Italia e fuori d'Italia, ma anche e soprattutto i discendenti degli Italiani, gli italofoni e gli italofili: una comunità globale stimata attorno ai 250 milioni di persone nel mondo, alle quali la globalizzazione conferisce significati e potenzialità nuove e sono ora chiamati a dare un senso alla loro aggregazione"'.

Da questa visione originale risulta che l'italianità si iscriverebbe nella resa concettuale offerta dall'italicità, senza togliere all'una o all'altra una distintiva dose di autonomia semantica e funzionale. È importante tenerlo a mente sviluppando le ulteriori riflessioni sull'italianità, che in fin dei conti non è riservata specificamente agli Italiani, ma ispira e diventa uno scopo sociale di un largo pubblico che l'alimenta di idee e di una specie di solidarietà etnicoculturale globalizzata.

Quanto l'italianità, anche l'italicità si estende comunque su campi intellettuali anche molto ampi, ostacolando la stesura di una sua univoca definizione. L'italicità suggerisce un insieme di attori o partecipanti (spesso attivi in questo contesto solo occasionalmente), ma secondo noi non sono solamente persone fisiche; anzi in molti casi sono intere organizzazioni o istituzioni (p.es. i corsi d'italianistica nelle università di vari paesi), ma anche

1 www.globusetlocus.org/attivita/popoli_glocal.kl [27/10/2020]. 
un tipo di patrimonio culturale, letterario e scientifico prodotto fuori dall'Italia e sull'Italia, la sua cultura, la lingua, la società, ecc.

L'italicità è poi uno spazio di impressioni, emozioni, giudici soggettivi che permettono di avvicinarsi all' italianità, ma nella maggioranza dei casi in modo superficiale, senza approfondimento ${ }^{2}$.

L'italicità è infine una pretesa di avere un'esperienza che spiega ai nostri occhi la passione verso l'italianità, in fondo un'esperienza senza radici né una continuazione, anche per le mancate opportunità di cui si dispone fuori dal contesto tipicamente italiano ${ }^{3}$.

Dunque, parlare di italianità, includendo anche l'italicità, ovvero l'italofilia ed anche l'italomania, è un'impresa alquanto complicata, vista proprio la complessità del soggetto, insita nel concetto di italianità. Un primo passo concreto per affrontare questa complessità, termine molto ricorrente, viene fatto proprio nel riconoscerla presente nel concetto di italianità in tutti i campi: a cominciare dalla varietà geografica dell'Italia come matrice prima della varietà socioculturale, storica e linguistica degli Italiani.

\section{Estraneità contro superficialità}

Per avere un quadro il più possibile veritiero e completo sul concetto di italianità si tratta in primo luogo di evitare di guardare alla realtà italiana, passata e presente, come la protagonista di Fantasticheria del Verga (1880), dalla parte sbagliata del binocolo, quella che riduce tutto ad un simpatico quadretto in cui diventa praticamente impossibile scorgere i particolari drammi e le semplici felicità quotidiane delle persone che pure fanno parte di quel quadretto. Quindi, dopo aver affermato la complessità del concetto di italianità, di certo conviene seguire il consiglio di Verga, di osservare da vicino le cose, analizzarle, cercare di capirne le origini:

Bisogna farci piccini anche noi, chiudere tutto l'orizzonte fra due zolle, e guardare col microscopio le piccole cause che fanno battere i piccoli cuori. Volete metterci un occhio anche voi, a cotesta lente? voi che guardate la vita dall'altro lato del cannocchiale? Lo spettacolo vi parrà strano, e perciò forse vi divertirà (Verga 1983: 43).

$2 \mathrm{Si} \mathrm{vedano} \mathrm{p.es.} \mathrm{le} \mathrm{reazioni} \mathrm{entusiastiche} \mathrm{riguardo} \mathrm{alla} \mathrm{cucina} \mathrm{italiana} \mathrm{nel} \mathrm{mondo}$ o una stereotipata convinzione che per comunicare e soddisfare gli Italiani nell'uso della loro lingua bastino tre-quattro parole di base o del tutto casuali.

3 Per considerazioni ulteriori sull' italicità, anche alla luce dell' italianità si vedano per. es. Bassetti (2008); Machetti (2010); Vedovelli (2008); Roić (a c.d. 2006). 
Tuttavia, l'idea del cannocchiale rovesciato ricorre anche in un altro autore siciliano, Luigi Pirandello (1993: 162), che attraverso il dottor Fileno, propone di applicare questa volta non tanto una visione superficiale quanto piuttosto distaccata della realtà con la "filosofia del lontano". In questa filosofia si trattava appunto di guardare alla realtà complessa e multiforme con un atteggiamento di estraneità, riuscendo a cogliere come assurde quelle situazioni che, da chi ci vive immerso da anni, ormai vengono considerate "normali".

È normale, per esempio, che a Napoli si usi il clacson delle auto con disinvoltura; è normale che qualcuno parcheggi in doppia fila per fermarsi a bersi un caffè in un bar; è normale la poca solerzia dei dipendenti statali nei confronti degli utenti, a meno che non si tratti di amici o parenti; è normale usare nella lingua italiana termini come "capofamiglia", "piuttosto che" con funzione disgiuntiva e i più recenti "runner e rider"; e tante altre cose sono considerate "normali", che magari affascinano, turbano o indignano il turista straniero, il purista della Crusca, l'italiano all'estero, insieme a tutti coloro che trovano infatti strane queste "normalità". Qualcuno potrà ribadire che è tutto frutto di un pregiudizio: che a Napoli i clacson vengono usati con moderazione e sempre secondo la legge; che non si è più visto qualcuno parcheggiare in doppia fila dai tempi del '68; che il personale amministrativo, secondo una recente ricerca statistica di un imprecisato Ente nazionale, risultano i più efficienti al mondo; che "capofamiglia" è una parola ormai in disuso e che "runner e rider" le usano esclusivamente i giornalisti di Rail e, infine, "piuttosto che" viene usato prevalentemente con il valore di "anziché".

Si riesce sempre a trovare qualcuno in Italia in grado dimostrare come vero un concetto e qualcun altro subito pronto a dimostrare vero il suo contrario. L'utilizzo che si può fare del cannocchiale rovesciato ce l'ha dimostrato. Tesi contraria: è sbagliato guardare alla realtà italiana come se fosse una bella cartolina illustrata, senza entrare nei particolari, senza parlare con la gente e vivere con loro dentro quella realtà, altrimenti si rischia di non capire cosa mai sia l'italianità. Tesi a favore: bisogna distaccarsi dalla realtà italiana per coglierne appieno la sua essenza: essere troppo coinvolti ci potrebbe rendere

4 In passato (fino al 1975), il capo della famiglia, ossia il marito (a cui per legge spettava la patria potestà) $\mathrm{o}$, in mancanza di questo, la madre vedova; in leggi speciali (tributarie, previdenziali, ecc.), la qualifica poteva spettare, in caso di inabilità dei genitori, ad altro familiare, per es. a uno dei figli. Con il nuovo diritto di famiglia tale qualifica viene meno, avendo $i$ coniugi parità di posizione sia nei riguardi reciproci sia nei confronti della prole, ed essendo la patria potestà sostituita dall'esercizio della potestà dei genitori (o potestà genitoriale). www.treccani.it/vocabolario/ capofamiglia/ [25/10/2020].

5 Rispettivamente in italiano: corridore, podista e fattorino. 
ciechi di fronte a situazioni che sono "assurde e anormali", anch'esse facenti parte dell'italianità. In realtà, a ben vedere, le due tesi non si escludono a vicenda, ma si integrano in un lavoro, che il concetto di italianità richiede, di analisi e sintesi. Dunque, per cercare di definire il concetto di italianità, occorre tenere a mente questa operazione di avvicinamento/allontanamento: cogliere allo stesso tempo il particolare e l'universale.

\section{Un Paese pieno di contraddizioni e di contrasti}

In cosa consisterà dunque questa "complessità" per definire l'italianità? Prima di tutto, ritornando all'aspetto geografico, la posizione stessa del Paese, nel mezzo del Mediterraneo, lo ha reso storicamente facile approdo per popoli e culture diverse: latini, etruschi, greci, arabi, goti, longobardi, normanni, ecc.

L'Italia risulta per la ragione suddetta un paese strabordante di storia, civiltà e cultura, ma tutto ciò contrasta con l'attuale tasso di analfabetismo funzionale, il più alto al mondo tra i paesi industrializzati ${ }^{6}$. Anzi si dirà di più. Il Paese che ha inventato il sistema bancario, che ha diffuso nel mondo termini come: banca, saldo, netto, valuta, girante, mercante, ecc.; che grazie alla sua intraprendenza imprenditoriale, l'industria manifatturiera, il famoso "made in Italy", fa ancora parte degli 8 paesi più industrializzati al mondo, ma allo stesso tempo è anche ai primi posti al mondo per il debito pubblico ${ }^{7}$ e attualmente con la crescita del PIL più bassa rispetto agli altri paesi dell'UE ${ }^{8}$. Per non far mancare nulla all'Italia, è anche il Paese con la più alta evasione fiscale in Europa e con il sistema fiscale più complicato?.

L'Italia è la terra dell'amor cortese e delle donne-angelo, della rima amorecuore-fiore, ma negli ultimi decenni con il più basso indice di natalità in Europa $^{10}$.

6 espresso.repubblica.it/inchieste/2017/03/07/news/analfabeti-funzionaliil-dramma-italiano-chi-sono-e-perche-il-nostro-paese-e-tra-i-peggiori-1.296854 $[11 / 11 / 2020]$.

7 www.money.it/debito-pubblico-piu-alto-classifica-paesi-Italia-FMI [11/11/2020].

8 www.rgs.mef.gov.it/VERSIONE-I/e_government/amministrazioni_pubbliche/igrue/PilloleInformative/economia_e_finanza/index.html?Prov=PILLOLE\#stat1 [11/11/2020].

9 www.corriere.it/dataroom-milena-gabanelli/tasse-siamo-davvero-paese-piu-tartassato-europa-iva-accise-cuneo-fiscale/f1515748-20ed-11ea-ad99-8e4d121df86f-va.shtml [11/11/2020].

10 www.ilsole24ore.com/art/culle-vuote-italia-maglia-nera-la-natalita-fortunache-ci-sono-migranti-ACVLLqX [11/11/2020]. 
L'Italia, possiede il $70 \%$ del patrimonio artistico mondiale ${ }^{11}$ ma anche il Paese che, destinando alla cultura solo lo $0.6 \%$ del $\mathrm{PIL}^{12}$, è agli ultimi posti in Europa.

L'Italia è la culla del diritto romano, del Corpus iuris civilis, ma anche la terra in cui sono sorte la mafia, la camorra, la sacra corona unita e infine l'ndrangheta, vale a dire la più potente organizzazione fuorilegge al mondo. Si badi bene, queste organizzazioni non sono un male passeggero, né tanto meno un male inevitabile, oppure un incidente di percorso e neanche una semplice avaria del sistema a cui si può facilmente mettere riparo. È qualcosa che il giudice Giovanni Falcone ormai considerava appartenente, per quanto la cosa possa non piacere, all'Italia e agli Italiani e quindi all' italianità: "Se vogliamo combattere efficacemente la mafia, non dobbiamo trasformarla in un mostro né pensare che sia una piovra o un cancro. Dobbiamo riconoscere che ci rassomiglia"13.

Vista così, da vicino e da lontano, questa Italia ricca di arte e di storia, illegale, familista, sempre più ignorante e povera sia finanziariamente che moralmente parlando, assomiglia molto tragicamente ancor di più a quel paese dei balocchi di cui parlava Collodi nel suo Pinocchio:

Dove vuoi trovare un paese più sano per noialtri ragazzi? Lì non vi sono scuole: lì non vi sono maestri: lì non vi sono libri. In quel paese benedetto non si studia mai. Il giovedì non si fa scuola: e ogni settimana è composta di sei giovedì e di una domenica. Figurati che le vacanze dell'autunno cominciano col primo di gennaio e finiscono coll'ultimo di dicembre. Ecco un paese, come piace veramente a me! Ecco come dovrebbero essere tutti i paesi civili!... - Ma come si passano le giornate nel «Paese dei balocchi»? - Si passano baloccandosi e divertendosi dalla mattina alla sera. La sera poi si va a letto, e la mattina dopo si ricomincia daccapo. Che te ne pare? ${ }^{14}$

Un Paese che, nonostante gli evidenti problemi, si è lasciato andare a partire dagli anni ' 80 alla vita mondana, al mondo colorato della televisione privata, composto di veline, calciatori, comici-politici e politici-comici dalla battuta facile, nonché una massa di personaggi di imprecisabile collocazione

11 www.usnews.com/media/best-countries/overall-rankings-2017.pdf $[11 / 11 / 2020]$.

12 ec.europa.eu/eurostat/statistics-explained/index.php?title=Culture_ statistics_-_government_expenditure_on_culture [11/11/2020].

13 In www.miur.gov.it/documents/20182/0/0522_Intervento+Porto+Civita vecchia.pdf/ef43bc47-6f2e-40f2-aa2d-6790c3ffcb7d?version $=1.0 \& \mathrm{t}=1527015082200$ [11/11/2020].

14 www.pinocchio.it/Download/Testo_ufficiale_LeAvventure_di_Pinocchio. $\operatorname{pdf}[11 / 11 / 2020]$. 
professionale, che allietanole serate dei telespettatori, sempre più teledipendenti e "incoscienti" ${ }^{15}$, tra i salotti televisivi dei talk-show, in un'atmosfera da "movida" serale della "Milano da bere", per poi scoprire un giorno che il principale artefice di questo mondo colorato, e allo stesso tempo una delle massime autorità dello Stato italiano, praticava il "Bunga-bunga", facendo toccare all'italianità uno dei punti moralmente più bassi degli ultimi decenni. Fa parte anche questo dell' italianitá? Leggendo Leopardi sembrerebbe di sì:

La vivacità del carattere italiano che fa loro preferire i piaceri degli spettacoli e gli altri diletti de'sensi a quelli più particolarmente propri dello spirito, e che gli spinge all'assoluto divertimento scompagnato da ogni fatica dell'animo e alla negligenza e pigrizia $^{16}$.

L'Italia è la terra dei perenni contrasti: Guelfi e Ghibellini, Don Camillo e Peppone, Monarchia e Repubblica, guardie e ladri, Montecchi e Capuleti, uomini e caporali, Nord e Sud, fascisti e partigiani, Cadorna e Diaz, terroni e polentoni, studenti e celerini, olio e burro, città e campagna, Bartali e Coppi, comunisti e democristiani, borghesi e proletari, pecorino e parmigiano, Fiat e Alfa Romeo, Modugno e Villa. La lista termina qui, in quanto potrebbe risultare, purtroppo, assai lunga.

Pensiamo sia chiaro a tutti che l'Italia è un paese geograficamente diversificato, con una storia intensa e a volte anche difficile da accettare; con una cultura stratificata e una lingua nazionale apparentemente recente ma con una solida letteratura. È forza di cose quindi che l'italianità risulti complessa, non può essere altrimenti. Cerchiamo quindi di fare un po' d'ordine.

\section{A cosa corrisponde l'italianità?}

Accade spesso, cercando tra dizionari diversi, che una parola abbia più di una definizione e questi molteplici approcci alla medesima parola li possiamo considerare come le diverse informazioni contenute in una carta d'identità. Ogni singolo elemento del documento rimanda sempre alla stessa persona, ma considerato singolarmente è insufficiente per definirne con certezza l'identità.

15 "...la televisione, comunque inchioda la gente... La televisione impigrisce e la paura diventa più una scusa, perché alle 9 di sera si rientra in casa e, poi, non si ha più voglia di uscire, perché c’è la televisione che ti fa passare quelle due e tre ore prima di andare a dormire" (Ginsborg 1998: 658).

16 Giacomo Leopardi, Discorso sopra lo stato presente dei costumi degl'Italiani, in www.liberliber.it/mediateca/libri/l/leopardi/discorso_sopra_lo_stato_etc/pdf/ discor_p.pdf, p. 7. [11/11/2020]. 
Allo stesso modo, ogni singola proposizione di ogni singola definizione che troviamo nei dizionari, pur riferendosi sempre alla stessa parola, tuttavia è insufficiente per fornircene una descrizione completa. Se continuiamo con il paragone e consideriamo che il documento d'identità non è equivalente totalmente alla persona viva in carne ed ossa, in quanto descrive solo alcuni aspetti di essa ma ne tralascia moltissimi altri, per es. il carattere, il numero di scarpe portato, la squadra del cuore, vizi e virtù, ecc. Similmente, gli elementi delle definizioni di certe parole, soprattutto di concezioni astratte, prese tutte insieme, non riusciranno mai a darcene una descrizione totale, ma inesorabilmente rimarrà taciuta una parte (grande o piccola che sia) di essa. La parola italianità ne fornisce un valido esempio attraverso le diverse definizioni trovate in alcuni dizionari:

- partecipazione al patrimonio di cultura e civiltà attribuito alla nazione italiana (Devoto-Oli 1989);

- indole, natura, qualità di italiano (Zingarelli 1989);

- L'essere conforme a ciò che si considera peculiarmente italiano o proprio degli Italiani nella lingua, nell'indole, nel costume, nella cultura, nella civiltà, e sim. 2. Più com., l'essere e il sentirsi italiano; appartenenza alla civiltà, alla storia, alla cultura e alla lingua italiana, e soprattutto la coscienza di questa appartenenza ${ }^{17}$;

- Complesso di caratteri storici e culturali che connotano fortemente il costume e la psicologia degli italiani; 2. Senso di appartenenza all'Italia; 3. Conformità di una espressione linguistica all'italiano codificato dalle grammatiche ${ }^{18}$;

- l'insieme dei caratteri storici e culturali che connotano il costume, la cultura, la civiltà e l'indole degli italiani; 2 . qualità di chi, di ciò che è italiano; l'essere, il sentirsi italiano; senso di appartenenza all'Italia ${ }^{19}$.

Come possiamo notare, le definizioni nel loro insieme presentano dei punti in comune, alcune invece riportano concezioni completamente diverse, comprendendo o escludendo diversi elementi o attributi, che dovrebbero far parte dell'italianità. Ciò che risalta in molte definizioni è il concetto di "appartenenza" che si riferisce a tre elementi in particolare: Italia, Italiani e lingua italiana.

Riguardo al primo elemento, una difficoltà si pone soprattutto se pensiamo all' italianità come tutto ciò che appartiene all'Italia, non solo come "espressione

17 www.treccani.it/vocabolario/italianita/ [25/10/2020].

18 dizionari.corriere.it/dizionario_italiano/I/italianita.shtml [25/10/2020].

19 www.dizionario-italiano.it/dizionario-italiano.php?parola=italianit\%C3\%A0 $[25 / 10 / 2020]$. 
geografica" ${ }^{20}$, ma come nazione. E qui sorge il primo problema, connesso anche alla datazione dell'italianità: a quale momento storico dobbiamo riferirci per definire l'Italia come nazione?

A questo punto ci sono due definizioni di nazione da prendere in considerazione:

1. complesso di persone che hanno origine, lingua, storia e cultura in comune, e che hanno coscienza di questa unità;

2. unità politica realizzata in uno stato nazionale.

Seguendo la prima definizione sopra riportata, potremmo parlare di nazione in territorio italiano già a partire dal periodo storico dell'impero di Ottaviano Augusto, in cui l'Italia intera era compresa in un'unica provincia senatoria. Tuttavia, visto che la lingua comune era allora il latino e la cultura era prevalentemente romana, in questo caso più che di italianità dovremmo piuttosto parlare di latinità o romanità.

Mentre la seconda definizione di nazione, ci costringerebbe a datare l'italianità ufficialmente al 1861, quindi all'Unità d'Italia e a tutto ciò che segue questa data. Tuttavia, se seguiamo pedissequamente questa alternativa, dobbiamo escludere dall'italianità: Dante, Boccaccio, Petrarca, tutto l'Umanesimo e il Rinascimento!

Una soluzione, a questo punto, potrebbe essere quella di considerare l'italianità non tanto ciò che appartiene all'Italia, intesa come nazione politica, quanto piuttosto ciò che le appartiene come nazione linguistica. Una nazione linguistica con una gestazione lunga ed una nascita ufficiale ${ }^{21}$ intorno la fine del $\mathrm{X}$ sec. Crediamo che questa sia una datazione abbastanza convincente, dalla quale possiamo cominciare a parlare di italianità, includendo in essa anche gli aspetti che contraddicono il concetto di nazione: la mancata coesione politica, territoriale e tantomeno culturale. Basti pensare soltanto alla turbolente realtà politica di quel periodo storico altamente frammentata, che si presentava pressappoco in questo modo: Longobardi, Bizantini, Arabi, Germani (Sud d'Italia); il Regno d'Italia (Piemonte, Lombardia, Liguria e Toscana) conteso tra il marchese d'Ivrea e Ottone I; territori soggetti alla Chiesa di Roma (Marche e Lazio); varie Signorie fondiarie sparse che danno luogo al fenomeno dell'incastellamento e alla nascita delle prime civitates (oltre i vecchi centri urbani di epoca romana, si formano agglomerati urbani intorno al palazzo del vescovo, alle abbazie e monasteri). Queste nuove realtà urbane provocano di fatto la fine dell'alto Medioevo e la diffusione e l'estensione dei primi traffici commerciali:

20 Frase attribuita a Klemens von Metternich.

21 Con i placiti cassinesi, o meglio conosciuti come: Carta Capuana. Vedi: www. cdsconlus.it/index.php/2016/09/26/alle-origini-della-lingua-italiana-il-placitocassinese-del-960-una-suggestiva-rilettura/ [13.11.2020]. 
Il fatto di "ammassare uomini" di "congregarli insieme" nel castrum o nella civitas fa sì che dal tipico insediamento sparso e dissociato dell'alto Medioevo si passi a un insediamento concentrato e finalizzato, con tutte le conseguenze che ne derivano su tutti i piani (Cracco 1986: 104).

È proprio quella frammentazione politica e culturale intorno all'anno Mille, da cui scaturisce quella 'complessità', che costituisce una costante, una sorta di marchio di fabbrica, se si vuole anche una tara ereditaria o perfino punto di forza dell'italianità. I placiti cassinesi sono "la testimonianza di quel risveglio, di quel rinnovamento che si nota nella penisola verso il Mille" (Migliorini 2001: 84); in cui germoglia, timidamente dal tardo latino, la lingua italiana, in forma di Volgare, che si afferma dapprima in toscana nel Medioevo. In seguito, rinforzando le sue radici nella letteratura riesce a estendere lentamente i suoi rami alle altre regioni italiane, imponendosi nel tempo a divenire una lingua non solo scritta ma anche parlata. Infine, possiamo considerare, dopo oltre mille anni, che tuttora la lingua italiana è divenuta uno dei pochi simboli di unità di tutti gli Italiani.

Alla luce di questo ragionamento siamo ora in grado di presentare una definizione di italianità maggiormente completa, rispetto a quelle presentate all'inizio di questo paragrafo.

L'italianità è il complesso di varietà (geografiche, culturali, storiche e civili) legate, appartenenti e riferentesi al territorio italiano, ai suoi abitanti e alla lingua italiana a partire dal secolo $\mathrm{X}$ fino ai giorni nostri.

\section{Il peso dell' italianità}

Immaginiamo di avere una bilancia su cui sia possibile pesare le parole. Su un piatto abbiamo la parola italianità che di certo, come già si è intuito, dalla definizione da noi appena fornita sopra, avrà un suo valore, un certo peso. Cosa ci metteremo nell'altro per equiparare quel peso? Ovvero, quali parole, personaggi, fatti storici, tradizioni popolari, immagini, emozioni, monumenti, quadri, cibi ecc. dovremmo porre sullaltro piatto per tentare di arrivare a una qualche equivalenza con la parola italianità? Pur sapendo, visto la complessità di cui si parlava prima, che questa equivalenza è praticamente irraggiungibile, senza rischiare di proporre solo degli stereotipi e di trascurare magari elementi che pure fanno parte dell' italianità. E immaginiamo noi stessi in una fila interminabile di persone, provenienti dal passato e dal presente, davanti a quella bilancia, tra cui Italiani DOC, Italiani all'estero, emigranti, figli e nipoti di emigranti, e tantissimi stranieri, tra cui quelli che vissero o vivono tuttora in Italia, che visitarono o hanno visitato l'Italia di recente, gli italomani e infine gli italici. Ognuno che tiene in mano una propria lista di attributi di italianità da porre su quel piatto. 
Riflettiamo per un momento: cosa conterrebbe la nostra lista personale? Ecco, anche i contributi, originali e non convenzionali, presenti in questo libro, potrebbero esser aggiunti su quel piatto della bilancia. E ben vengano in futuro anche degli altri! Perché, proprio grazie a questi diversi contributi, noi ci avviciniamo al concetto di italianità e possiamo fornirne una definizione maggiormente completa: il più possibile equivalente ed equilibrata. Perché, pensiamo alle conseguenze che ci sarebbero se qualcuno facesse sparire d'un colpo tutte le testimonianze sull' italianità e ne lasciasse intatta solo una, tipo:

Conosci tu il paese dove fioriscono i limoni?

Brillano tra le foglie cupe le arance d'oro,

Una brezza lieve dal cielo azzurro spira,

Il mirto è immobile, alto è l'alloro!

Lo conosci tu?

Laggiù! Laggiù!

$O$ amato mio, con te vorrei andare! $!^{22}$

Goethe presenta in questi versi una bella Italia solare. Difatti sono stati scelti proprio per proporre la discussione scientifica sull' italianità di cui qui presentiamo i frutti. Tuttavia, qualcuno, non sapendo che il poeta tedesco parla proprio dell'Italia, potrebbe attribuire le suddette parole anche alla Grecia o alla Spagna. Ma se d'Italia si tratta, allora si esclude, attraverso questa immagine, una buona parte di regioni italiane, in cui di arance e limoni non c'è nemmeno l'ombra. Di nuovo si ripresenta il tema della complessità quando si parla di italianità, contenente anche delle esclusioni al livello metodologico di una definizione vincolante: manca sempre qualcosa per dare un quadro unitario e in particolare nella suddetta immagine piena di sole, calore e colori mancano proprio gli "Italiani".

\section{Chi sono gli Italiani?}

In effetti, dopo aver parlato dell'Italia come nazione linguistica, e molto di sfuggita della lingua italiana, a cui bisognerebbe dedicare maggior spazio di quanto ne sia stato reso disponibile per questo contributo; occorre occuparci anche degli "Italiani", uno degli elementi certamente essenziale dell'italianità, ma che secondo Massimo D’Azeglio, raggiunta l'Unità nel 1861, erano ancora da "fare" o meglio da "rifare":

I più pericolosi nemici d'Italia non sono gli Austriaci, sono gli Italiani.

E perché? Per la ragione che gl'Italiani hanno voluto far un'Italia nuova,

22 Traduzione di Anita Rho e Emilio Castellani in J. W. Goethe, Wilhelm Meister. Gli anni di apprendistato, Adelphi, Milano, 1974. 
e loro rimanere gl'Italiani vecchi di prima, colle dappocaggini e le miserie morali che furono ab antico il loro retaggio [... Il primo bisogno d'Italia è che si formino Italiani dotati d'alti e forti caratteri. E pur troppo si va ogni giorno più verso il polo opposto: pur troppo s'è fatta l'Italia, ma non si fanno gl'Italiani (Del Boca 2008: 38).

Probabilmente frugando in quel piatto della bilancia, in cui sono presenti le equivalenze di italianità, potremmo trovare molte testimonianze su di essi, che ripetono, come quella appena riportata sopra, un triste ritornello:

Terra di infanti, affamati, corrotti, governanti impiegati di agrari, prefetti codini, avvocatucci unti di brillantina e i piedi sporchi, funzionari liberali carogne come gli zii bigotti, una caserma, un seminario, una spiaggia libera, un casino!

(Pasolini 2014: 129)

C’è qualcuno che possa dire che Pasolini non parli proprio di Italiani in questi versi? Purtroppo, in questi versi, sono descritti alcuni tipi di Italiani ma, per fortuna, anche questa è solo una parte della verità. Ma se ci pensiamo bene, le testimonianze negative di Italiani sugli Italiani sono sia antiche che contemporanee:

Vano error vi lusinga:

poco vedete, et parvi veder molto, ché $n$ cor venale amor cercate ofede.

(Petrarca 1989: 76, canzone CXXVII, versi 23-25)

\section{Oppure:}

Questo paese è devastato dal dolore...

Ma non vi danno un po' di dispiacere

quei corpi in terra senza più calore?

Non cambierà, non cambierà

non cambierà, forse cambierà. ${ }^{23}$

Si direbbe il caso che Petrarca e Battiato non siano interessati, parlando, o meglio cantando, della loro Italia, ad arance e limoni: emergerebbe anzi che gli Italiani, in qualsiasi periodo si tratti, quando si rivolgono ai loro compatrioti, non sono molto delicati. Sono piuttosto ironici, cinici, sprezzanti, implacabili,

23 Brano dalla canzone: Povera Patria di Franco Battiato, contenuta nell'album: Come un cammello in una grondaia del 1991. 
decisamente pessimisti; li accusano spesso di immobilismo, parassitismo, soprattutto se vengono messi a confronto con le glorie passate:

O patria mia, vedo le mura e gli archi

E le colonne e i simulacri e l'erme

Torri degli avi nostri,

Ma la gloria non vedo,

Non vedo il lauro e il ferro ond'eran carchi

I nostri padri antichi. Or fatta inerme

Nuda la fronte e nudo il petto mostri,

Oimè quante ferite,

Che lividor, che sangue! oh qual ti veggio,

Formesissima donna! ${ }^{24}$

\section{Oppure:}

Ahi serva Italia, di dolore ostello, nave sanza nocchiere in gran tempesta, non donna di provincie, ma bordello!

(Dante 1988: 104, Pg VI 76-78)

Di nuovo, attraverso Leopardi e Dante, emerge il tema dei contrasti: amore e odio, luminoso passato e oscuro presente. Amore verso le bellezze del territorio, i monumenti e la gloria che passò; odio verso i suoi abitanti a loro contemporanei e rammarico per la miseria in cui è caduto inesorabilmente il Paese. Stendhal a questo riguardo disse una frase divenuta celebre: "Un popolo di giganti e di eroi è morto nel 1530 ed è stato rimpiazzato da un popolo di pigmei" 25 .

Lo scrittore francese non è il solo, ci sono una serie di stranieri autorevoli del passato che durante i loro viaggi diplomatici oppure durante il loro Grand Tour, non avevano raccolto degli abitanti del Bel Paese un'opinione molto positiva:

Gli italiani erano definiti, tout court, pigri, scansafatiche, indifferenti. $\mathrm{E}$ inoltre ignoranti, creduloni, baciapile, papisti. E ancora: inaffidabili, voltagabbana, servili, imbelli. E anche insensibili a tutti gli ammonimenti, a tutti gli insulti, persino alle pedate (Del Boca 2008: 11).

24 Incipit della poesia: All'Italia di Giacomo Leopardi, da: I Canti, Sansoni Editore, Firenze, 1985, p. 13.

25 www.ilfoglio.it/cultura/2016/04/24/news/gli-italiani-di-stendhal-unpopolo-di-antichi-giganti-divenuti-pigmei-95282/ [13/11/2020]. 
Qualcuno dirà che le testimonianze presentate sopra provengono esclusivamente da Italiani o stranieri che covavano senso di superiorità, rancore, rabbia, senso di impotenza, ecc.; e che questi sentimenti negativi erano perlopiù indirizzati alla classe dominante, al potere, non tanto contro i poveri cristi e madonne disseminati tra i sassi di Matera, i latifondi siciliani, l'agro romano, le pianure venete e le valli alpine, ecc.

Di certo, non appartenevano a quei milioni di Italiani che, già a partire dalla fine del XIX secolo e per una buona metà del secolo seguente, fuggirono per sempre da questa amara terra, "amara e bella" ${ }^{26}$, non solo per cercare fortuna altrove, ma semplicemente per sopravvivere. Questi giudizi non sono neanche riferibili a quelle centinaia di migliaia di soldati italiani che morirono tra le trincee e le gallerie alpine scavate quasi a mani nude durante la grande guerra.

Piuttosto, quei giudizi, quasi fossero un monito per il futuro, forse possono avere un destinatario in quei milioni di Italiani, di tutte le classi sociali, che dopo l'esperienza disastrosa della I guerra mondiale, che pur avrebbe dovuto loro insegnare qualcosa, si gettarono entusiasti e ubbidienti tra le braccia di Mussolini: "Insomma, più adatta agli italiani appariva una dittatura, un governo forte e autoritario, capace di imporre disciplina a un popolo immaturo, inconsapevole dei propri diritti, non preparato all'esercizio delle libertà" (Colarizi 2000: 196).

La maturità e la consapevolezza furono poi raggiunte a caro prezzo, dopo aver fatto precipitare tutto il Paese in una immane catastrofe. E solo grazie alle migliaia di partigiane e partigiani, che gli Italiani, rappresentati da Alcide De Gasperi, hanno potuto guardare negli occhi i rappresentati delle Nazioni vincitrici alla conferenza di Parigi del 10 agosto del 1946:

Prendendo la parola in questo consesso mondiale, sento che tutto, tranne la Vostra personale cortesia, è contro di me, e soprattutto la mia qualifica di ex nemico, che mi fa considerare come imputato, e l'essere citato qui dopo che i più influenti di voi hanno già formulato le loro conclusioni in una lunga e faticosa elaborazione ${ }^{27}$.

Le complessità associate all'italianità vengono meno, quando arriva il momento per gli Italiani di essere uniti, con il 'Me' di De Gasperi che si riconosce "ex-nemico" e "imputato" e che si contrappone al becero e distruttivo "Me ne frego!" fascista, sopportando pazientemente le conseguenze per le azioni

26 Dalla celebre canzone di Domenico Modugno: Amara terra mia. Canzone che ripropone i versi di un canto abruzzese dei primi del '900: "Addije, addije amore". it.wikipedia.org/wiki/Amara_terra_mia [25/10/2020].

27 www.youtube.com/watch $\overline{\mathrm{v}}=\mathrm{pBaPZT}$ QX9E\&ab_channel=IstitutoLuce Cinecitt\%C3\%A0 [13/11/2020]. 
vergognose del Ventennio. Il 1946, l'anno dell'Assemblea Costituente, della nascita della Repubblica Italiana, grazie anche al voto concesso finalmente alle donne, e infine l'anno della Conferenza di Parigi, rappresenta una delle poche volte in cui gli Italiani hanno dato il meglio della loro italianità.

\section{Un'italianità oltre i pregiudizi e i falsi miti}

Dunque, siamo passati da una bella Italia soleggiata con i versi goethiani, tentando il difficile compito di voler dare una definizione di italianità, e ci siamo trovati a volte sotto un cielo cupo senza stelle, nel bel mezzo di una terra abitata da un popolo descritto spesso come disunito, insensibile, parassita. Occorre attraversare questa terra con gli occhi ben aperti, non c’è altra via, per giungere ad argomenti più sereni sull'italianità. Certamente è una scelta che possiamo volendo scantonare, limitandoci a starcene in una sorta di limbo ad ammirare il cielo azzurro, sole, il mare, senza dimenticare le arance e limoni, che con i loro colori vivi di certo quasi accecano la vista. Tuttavia, si rischia, in questo modo, di essere solo come dei pittori ciechi o cantastorie muti.

Chi vive o ha vissuto in Italia sa che ci sono in questo Paese dolori antichi e nuovi che si mescolano e permangono; ingiustizie e divisioni del passato che, non risolte, ritornano puntualmente nel presente. Ma conoscere e far conoscere tutto questo, anche se suscita rabbia e vergogna, e il saper raccontare di questi dolori ed ingiustizie è anche una parte consistente, vitale e positiva, dell'italianità.

Sì, perché possiamo addebitare agli Italiani molti difetti, ma di certo non di accusarli di essere degli ipocriti, oppure dei beati sempliciotti, nonché essere soggetti a sentimenti di superiorità nei confronti di altri popoli, non dopo aver vissuto fatti così terribili, di cui solo alcuni, accennati sopra.

Come abbiamo già visto nelle varie citazioni, gli Italiani e autorevoli osservatori stranieri, del passato e del presente, attraverso la prosa, la poesia, le canzoni popolari e quelle d'autore, i discorsi di alcuni politici, hanno cercato di raccontare, agli Italiani stessi, la realtà osservata da vicino e da lontano, senza nascondere nulla, se non altro per un'esigenza di verità:

Forse si stimerà che le cose nostre sieno più note a un italiano che non sono e non sarebbero a uno straniero, e finalmente se questi non dee risparmiare il nostro amor proprio con danno della verità, perché dovrò io parlare in cerimonia alla mia propria nazione, cioè quasi alla mia famiglia e a' miei fratelli $?^{28}$

28 Leopardi, op. cit., p. 5. 
In nome della sincerità e dell'umiltà, anche il cinema, con il neorealismo italiano, ha scaraventato sul grande schermo la cruda realtà del dopoguerra a chi credeva che si potesse guardare sognanti solo a un'Italia dai telefoni bianchi. Attori come Totò, Eduardo, Alberto Sordi, Anna Magnani e tanti altri hanno mostrato al grande pubblico, ridendo castigat mores, le grandezze e allo stesso tempo le miserie degli Italiani.

I cantautori come De André, Guccini, De Gregori ed altri hanno messo in musica verso la fine degli anni ' 60 i loro versi duri, che stridevano alle orecchie di quegli Italiani troppo abituati a bearsi soltanto di gorgheggi d'amore, storie sfortunate, cuori infranti, ecc.; in una musica fin troppo "leggera" che si rifaceva, come incantata, a certi modelli inglesi o americani, cercando di dimenticare il passato, senza occuparsi quasi per nulla di politica e tanto meno dei problemi sociali, in un periodo in cui il boom economico era ormai agli sgoccioli.

I libri, grezzi come mattoni, di autori come Pasolini, Pavese, Silone, Carlo Levi, Calvino, e ultimamente, Saviano, ecc., hanno raccontato agli Italiani, e al mondo intero, del dramma degli ultimi, i dimenticati, i fragili, dei "brutti, sporchi e cattivi ${ }^{29}$ " delle baraccopoli, delle anonime periferie cittadine. Tuttora presenti in questo Bel Paese.

Far conoscere e raccontare anche questa scomoda verità sull'Italia e gli Italiani, agli stessi Italiani e agli stranieri è come una sorta di Purgatorio: l'unico modo per poter giungere a quella tanto agognata equivalenza del concetto di italianità. Se tutti, Italiani e resto del mondo, tenessero in conto, di guardare alla realtà italiana, considerata in tutte le sue possibili varianti e varietà, analizzandola e sintetizzandola con partecipazione e estraneità, mentre si accingono a stilare la loro lista da porre sul suddetto piatto della bilancia. Forse, solo allora, sarà possibile apprezzare, godere pienamente e finalmente anche dei lati positivi dell'italianità. Solo riconoscendo e ammettendo le proprie grandezze e limiti, i propri errori e pregi, gli Italiani possono ritrovarsi finalmente uniti, non tanto per il fatto di essere semplicemente donne e uomini, quanto piuttosto, quello di ritrovarsi come sorelle e fratelli, figlie e figli di uno stesso, unico, Paese:

Fratelli d'Italia

L'Italia s'è desta [...]

Noi siamo da secoli

Calpesti, derisi,

Perché non siam popolo,

Perché siam divisi.

29 Titolo di un film di Ettore Scola del 1976. 


\section{Bibliografia}

Alighieri, Dante (1988). Divina Commedia: Inferno: Canto XXVI, Firenze, Le Monnier.

Colarizi, Simona (2000). Storia del Novecento italiano, Milano, BUR.

Cracco, Giorgio (1986). Il Medioevo, Torino, Sei.

Del Boca, Angelo (2008). Italiani, brava gente?, Vicenza, Neri Pozza.

Devoto, Giacomo e Oli, Gian Carlo (1989). Dizionario della lingua italiana, Firenze, Le Monnier. Ginsborg, Paul (1998). Storia d'Italia 1943-1996, Torino, Einaudi.

Goethe, Johann Wolfgang (1974). Wilhelm Meister. Gli anni di apprendistato, traduzione di Anita Rho e Emilio Castellani, Milano, Adelphi.

Leopardi, Giacomo (1824). Discorso sopra lo stato presente dei costumi degl'Italiani (vedi sitografia).

Leopardi, Giacomo (1985). All'Italia, da: I Canti, Firenze, Sansoni Editore.

Pasolini, Pier Paolo (2014). "Alla mia nazione”, [in] La religione del mio tempo, Milano, Garzanti.

Petrarca, Francesco (1989). Canzone CXXVII: Italia mia, benché 'l parlar sia indarno, [in] Rerum Vulgarium Fragmenta, alias il Canzoniere, Torino, Nuova Universale Einaudi.

Pirandello, Luigi (1993). La tragedia di un personaggio, dalla raccolta L'uomo solo (1922), vol. 2 , Roma, Newton.

Verga, Giovanni (1983). "Fantasticheria”, [in] Novelle, Novara, Epidem.

Zingarelli, Nicola (1989). Vocabolario della lingua italiana, XI edizione, Bologna, Zanichelli.

\section{Sitografia}

dizionari.corriere.it/dizionario_italiano/I/italianita.shtml [25/10/2020].

ec.europa.eu/eurostat/statistics-explained/index.php?title=Culture_statistics government_expenditure_on_culture [11/11/2020].

espresso.repubblica.it/inchieste/2017/03/07/news/analfabeti-funzionali-il-drammaitaliano-chi-sono-e-perche-il-nostro-paese-e-tra-i-peggiori-1.296854 [11/11/2020].

it.wikipedia.org/wiki/Amara_terra_mia. [25/10/2020].

www.cdsconlus.it/index.php/2016/09/26/alle-origini-della-lingua-italiana-il-placitocassinese-del-960-una-suggestiva-rilettura/ [13/11/2020].

www.corriere.it/dataroom-milena-gabanelli/tasse-siamo-davvero-paese-piu-tartassatoeuropa-iva-accise-cuneo-fiscale/f1515748-20ed-11 ea-ad99-8e4d121 df86f-va.shtml, $[11 / 11 / 2020]$.

www.democraticicristiani.com/vecchiosito/documenti/degasperi3.html [13/11/2020]. www.dizionario-italiano.it/dizionario-italiano.php?parola=italianit\%C3\%A0 [25/10/2020]. www.globusetlocus.org/attivita/popoli_glocal.kl [27/10/2020].

www.ilfoglio.it/cultura/2016/04/24/news/gli-italiani-di-stendhal-un-popolo-di-antichigiganti-divenuti-pigmei-95282/ [13/11/2020].

www.ilsole24ore.com/art/culle-vuote-italia-maglia-nera-la-natalita-fortuna-che-ci-sono-migranti-ACVLLqX [11/11/2020].

www.liberliber.it/mediateca/libri/l/leopardi/discorso_sopra_lo_stato_etc/pdf/discor_p. pdf $[27 / 10 / 2020]$.

www.miur.gov.it/documents/20182/0/0522_Intervento+Porto+Civitavecchia. $\mathrm{pdf} / \mathrm{ef} 43 \mathrm{bc} 47-6 \mathrm{f}-2 \mathrm{e}-40 \mathrm{f} 2$-aa2 $\mathrm{d}-6790 \mathrm{c} 3 \mathrm{ffcb} 7 \mathrm{~d}$ ? version $=1.0 \& \mathrm{t}=1527015082200$ $[11 / 11 / 2020]$. 
www.money.it/debito-pubblico-piu-alto-classifica-paesi-Italia-FMI, [11/11/2020].

www.pinocchio.it/Download/Testo_ufficiale_LeAvventure_di_Pinocchio.pdf $[11 / 11 / 2020]$.

www.rgs.mef.gov.it/VERSIONE-I/e_government/amministrazioni_pubbliche/igrue/

Pillole-Informative/economia_e_finanza/index.html?Prov=PILLOLE\#stat 1 , $[11 / 11 / 2020]$.

www.treccani.it/vocabolario/capofamiglia/ [25/10/2020].

www.treccani.it/vocabolario/italianita/ [25/10/2020].

www.usnews.com/media/best-countries/overall-rankings-2017.pdf [11/11/2020].

www.youtube.com/watch?v=pBaPZT_QX9E\&ab_channel=IstitutoLuceCinecitt\%C3\%A0 $[13 / 11 / 2020]$. 